\title{
Nuclear effects on tetraquark production by double parton scat- tering
}

\author{
Carvalho, F. ${ }^{1, a}$ and Navarra, F. S. ${ }^{2}$ \\ ${ }^{1}$ Departamento de Ciências Exatas e da Terra, Universidade Federal de São Paulo,Campus Diadema. Rua \\ Prof. Artur Riedel, 275, Jd. Eldorado, 09972-270, Diadema, SP, Brazil \\ ${ }^{2}$ Instituto de Física, Universidade de São Paulo, C.P. 66318, 05315-970 São Paulo, SP, Brazil \\ second here
}

\begin{abstract}
In this work we study the nuclear effects in exotic meson production. We estimate the total cross section as a function of the energy for $p P b$ scattering using a version of the color evaporation model (CEM) adapted to Double Parton Scattering (DPS). We fond that the cross section grows significantly with the atomic number, indicating that the hypothesis of tetraquark states can be tested in pA collisions at LHC.
\end{abstract}

\section{Introduction}

Over the last years many new exotic mesons have been measured in particle acelerators. In 2003, the Belle collaboration measured the X(3872) meson, which was later confirmed by CDF, D0, BABAR and $\mathrm{LHCb}$. Recently, the $\mathrm{LHCb}$ collaboration has masured its quantum numbers and these results suggest that its structure can be described by either a meson molecule, a tetraquark state or a hybrid state. In the molecular approach the estimated cross section is about two orders of magnitude smaller than the measured one, which suggests that the tetraquark could be more appropriated. It has been shown in ref. [3] that it is extremely difficult to produce molecules in pp collisions. Besides, the LHC has already performed measurements of $p A$ collisions at energies of $\sqrt{s}=7 \mathrm{TeV}$ and results obtained at $13 \mathrm{TeV}$ may be available next year. Motivated by the reasons above we have studied the $\mathrm{X}(3872)$ production in $p p$ in a previous work. We have also made an extension of the model to estimate the T4c cross section, an "all charm" tatraquark state. In this work we apply the same formalism to calculate the exotic meson production cross section in $p A$ collisions considering the tetraquark structure.

The $T_{4 c}$ state was first discussed long time ago by Iwasaky [4]. In the eighties and early nineties, many authors [5-8] addressed the subject arriving at different conclusions concerning the existence of a $c \bar{c} c \bar{c}$ bound state. More recently, with the revival of charmonium spectroscopy, Lloyd and Vary [9] investigated the four-body $c \bar{c} c \bar{c}$ system obtaining several close-lying bound states. They found that deeply bound $(\simeq 100 \mathrm{MeV})$ states may exist with masses around $6 \mathrm{GeV}$. In Ref. [10] the existence of $c \bar{c} c \bar{c}$ states was discussed in the framework of the hyperspherical harmonic formalism. The results suggested the possible existence of three four-quark bound states with quantum numbers $0^{+-}, 2^{+-}$and $2^{++}$and masses of the order of $6.50,6.65$, and $6.22 \mathrm{GeV}$. More recently, using the Bethe-Salpeter approach, the authors of Ref. [11] found an all-charm tetraquark with $J^{P C}=0^{++}$and mass $M_{T_{4 c}}=$

\footnotetext{
a e-mail: babi.usp@gmail.com
} 
$5.3 \pm 0.5 \mathrm{GeV}$. This mass is considerably lower than the $6.0 \mathrm{GeV}$ obtained in the previous model calculations $[4,9]$. It is also much lower than the $2 \eta_{c}$ threshold. Potential decay channels into D mesons and pairs of light mesons necessarily involve internal gluon lines. The resulting decay width may therefore be rather small. On the other hand, preliminary lattice QCD calculations [12, 13] seem to disfavor the existence of a deeply bound $c \bar{c} c \bar{c}$ state, being more compatible with a loosely bound $2 \eta_{c}$ molecular state. In the previous works $[14,15] T_{4 c}$ production was studied in single parton scattering (SPS) formalism, where events with four heavy quarks can be treated as a particular case of $\alpha_{s}^{2}$ correction to the standard single gluon-gluon scattering, in which an extra $c \bar{c}$ pair is produced, i.e., the process $g g \rightarrow c \bar{c} c \bar{c}$. Another possible way to produce $c \bar{c} c \bar{c}$ is by two independent leading order gluon-gluon scatterings, i.e. two times the reaction $g g \rightarrow c \bar{c}$. This is usually called double parton scattering (DPS) $[16,17]$.

Here, the relevant DPS process is the production of a $c-\bar{c}$ pair plus a light quark pair, $q-\bar{q}$, which will hadronize and form the $X(3872)$. Since DPS events are in the realm of perturbative physics, the light quark pair must be produced with large invariant mass and the final state $X(3872)$ will carry large transverse momentum. This seems to be appropriate to describe the CMS data [18], where the $X(3872)$ was observed with a transverse momentum lying in the range $10 \leq p_{T} \leq 25 \mathrm{GeV}$. In [19] it has been shown that DPS charm production is already comparable to SPS production at LHC energies. DPS grows faster with the energy because it is proportional to $g\left(x, \mu^{2}\right)^{4}$ while SPS is proportional to $g\left(x, \mu^{2}\right)^{2}$. Here $g\left(x, \mu^{2}\right)$ is the gluon density in the hadron as a function of the gluon fractional momentum $x$ and of the scale $\mu$ and it grows quickly with increasing collision energies. In the present work we shall consider the DPS events with the production of a $c \bar{c}$ and a light quark $q \bar{q}$ pair (X(3872)) and also with two $c \bar{c}$ pairs (T4c). If the two $q \bar{q}$ formed in the process bind together we have a tetraquark state. In the following section we present the mechanism of double parton scattering for hadron-hadron collisions and the extension of the CEM formalism to the production of tetraquarks.

\section{Tetraquarks Production Mechanism}

In this work we use the same hipothesis used in the ref. [19] for tetraquark production in pp interactions. As presented before, in the CEM formalism, the main ingredientthes are the gluon density in the hadron $g\left(x, \mu^{2}\right)$ and the partonic cross section $\sigma_{g g \rightarrow q \bar{q}}$.

For the SPS we have:

$$
\sigma_{M}=\int_{0}^{1} d x_{1} \int_{0}^{1} d x_{2} g\left(x_{1}, \mu^{2}\right) g\left(x_{2}, \mu^{2}\right) \sigma_{g_{1} g_{2} \rightarrow q \bar{q}} \times \Theta\left(M^{2}-4 m_{q}^{2}\right)
$$

where $g\left(x, \mu^{2}\right)$ is the gluonic distribution inside the corresponding hadron and $\sigma_{g_{1} g_{2} \rightarrow q \bar{q}}$ is the gluon-gluon $\rightarrow q \bar{q}$ elementary cross-section. The step function $\Theta\left(M^{2}-4 m_{q}^{2}\right)$ is to impose that the mass of the Meson must be bigger than a minimum mass which is set up equal to the mass of 2 quarks.

In our case, to produce two $q \bar{q}$ pairs, we use the double parton scattering, pictured in the Fig. 1.

Working with the usual CEM one-dimensional kinematics, the rapidities of the objects $M_{12}$ and $M_{34}$ are respectively:

$$
y_{12}=\frac{1}{2} \ln \frac{x_{1}}{x_{2}} \quad \text { and } \quad y_{34}=\frac{1}{2} \ln \frac{x_{3}}{x_{4}}
$$

and their invariant masses are

$$
M_{12}=\sqrt{x_{1} x_{2} s} \text { and } \quad M_{34}=\sqrt{x_{3} x_{4} s} .
$$




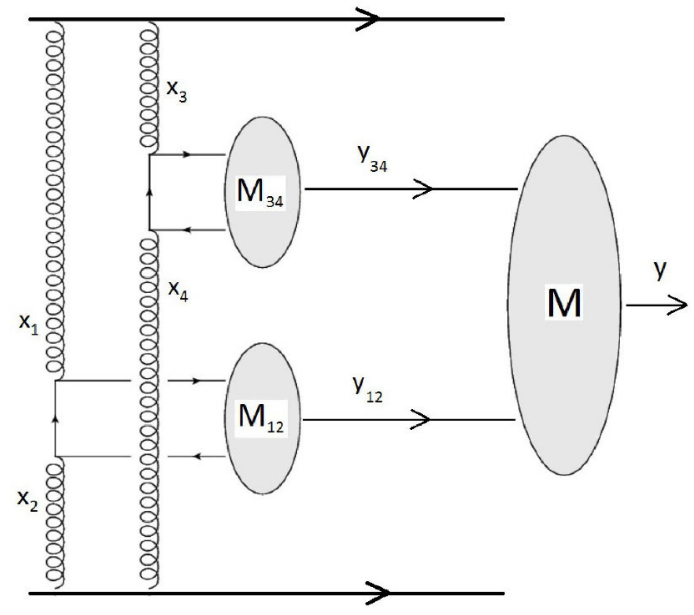

Figure 1. The gluons with odd (even) label come from the upper (lower) hadron, and carry momentum fraction $x_{i}$. The "gluon 1" scatters with the "gluon 2", making up a meson state with invariant mass $M_{12}$, and so on.

In terms of these variables and in the low $p_{T}$ regime, the invariant mass of the $c \bar{c} c \bar{c}$ system is then given by:

$$
M^{2}=M_{12}^{2}+M_{34}^{2}+2 M_{12} M_{34} \cosh \left(y_{12}-y_{34}\right) .
$$

The cosh function grows very rapidly with the argument and hence even a modest rapidity difference between the two clusters with $M_{12}$ and $M_{34}$ will significantly increase the value of $M$. We will then assume that both clusters move with equal rapidity, i.e. $y_{12}=y_{34}$, and become bound to each other, forming a system with mass:

$$
M=M_{12}+M_{34} \text {. }
$$

Finally, in order to produce the final tetraquark state with right mass, $M_{T}$, the cluster with mass $M$ emits or absorbs gluons carrying an energy $\Delta$, which will be discussed below. We have thus:

$$
M \pm \Delta=M_{T} .
$$

A remarkable difference between the standard CEM for charmonium production and the model developed here is in the role played by the limits of the integral over the squared invariant mass $M^{2}$. In the case of the usual $J / \psi$ production it goes from $\left(2 m_{c}\right)^{2}$ to $\left(2 m_{D}\right)^{2}$. This ensures that the $c-\bar{c}$ can never decay into open charm, not forming the charmonium state, because it does not have enough invariant mass. The case of the tetraquark $X(3872)$ is different. Suppose, for example, that we have the four-quark system with an invariant mass of $3740 \mathrm{MeV}$. While this system can only form the $X$ resonance by absorbing some gluons (carrying energy $\Delta$ ) from the target or from the projectile, it has sufficient mass to decay immediately into a $D^{+} D^{-}$pair and not form the resonance. Moreover, since the energy $\Delta$ is carried by an undefined number of gluons, this decay is not hindered by parity (or charge conjugation) conservation. Therefore, in our case, the integration over $M^{2}$ must be changed becoming more restrictive:

$$
\int_{\left(2 m_{c}\right)^{2}}^{\left(2 m_{D}\right)^{2}} d M^{2} \rightarrow \int_{\left(M_{T}-\Delta\right)^{2}}^{\left(M_{T}+\Delta\right)^{2}} d M^{2}
$$

where the left side refers to the usual CEM and the right side refers to tetraquark states. 


\section{The production cross section}

The cross section of the process shown in Fig. 1 can be calculated with the schematic DPS "pocket" formula:

$$
\sigma_{D P S} \propto \frac{\sigma_{S P S}^{12} \sigma_{S P S}^{34}}{\sigma_{e f f}}
$$

where $\sigma_{e f f} \simeq 15 \mathrm{mb}$ is a constant extracted from data analysis and $\sigma_{S P S}$ is the standard QCD parton model formula, i. e., the convolution of parton densities with partonic cross sections. To be more precise we expand the above formula showing the kinematical constraints introduced to study tetraquark production. It reads:

$$
\begin{aligned}
\sigma_{D P S} & =\frac{F_{T_{4 c}}}{\sigma_{e f f}}\left[\int_{0}^{1} d x_{1} \int_{0}^{1} d x_{2} g\left(x_{1}, \mu^{2}\right) g\left(x_{2}, \mu^{2}\right) \sigma_{g_{1} g_{2} \rightarrow c \bar{c}}\right] \\
& \times\left[\int_{0}^{1} d x_{3} \int_{0}^{1} d x_{4} g\left(x_{3}, \mu^{2}\right) g\left(x_{4}, \mu^{2}\right) \sigma_{g_{3} g_{4} \rightarrow c \bar{c}}\right] \\
& \times \Theta\left(1-x_{1}-x_{3}\right) \Theta\left(1-x_{2}-x_{4}\right) \Theta\left(M_{12}^{2}-4 m_{c}^{2}\right) \Theta\left(M_{34}^{2}-4 m_{c}^{2}\right) \\
& \times \delta\left(y_{34}-y_{12}\right)
\end{aligned}
$$

where $g\left(x, \mu^{2}\right)$ is the gluon distribution in the hadron with the gluon fractional momentum $x$ and at the factorization scale $\mu^{2}$ and $\sigma_{g g \rightarrow c \bar{c}}$ is the $g g \rightarrow c \bar{c}$ elementary cross-section. The step functions $\Theta\left(1-x_{1}-x_{3}\right)$ and $\Theta\left(1-x_{2}-x_{4}\right)$ enforce momentum conservation in the projectile and in the target. The step functions $\Theta\left(M_{12}^{2}-4 m_{c}^{2}\right)$ and $\Theta\left(M_{34}^{2}-4 m_{c}^{2}\right)$ guarantee that the invariant masses of the gluon pairs 12 and 34 are large enough to produce two charm quark pairs. The delta function implements the "binding condition" and $F_{T_{4 c}}$ is a constant, analogous to the one appearing in the CEM formula, which represents the probability of the four-quark system to evolve to a particular tetraquark state.

In the above formula, all the variables depend on the momentum fractions $x_{1} \ldots x_{4}$. Because of the delta function, we know that the two clusters shown in Fig. 1 are "flying together" and that they form a system with mass $M=M_{12}+M_{34}$, which can take any value. In order to improve our kinematical description of this bound state, we can impose constraints on the values of $M$, such as (7). This can be best done rewritting (9) and changing variables from $x_{1}, x_{2}, x_{3}$ and $x_{4}$ to $y_{12}, y_{34}, M_{12}$ and $M_{34}$. We obtain:

$$
\begin{aligned}
\sigma_{D P S} & =\frac{F_{T_{4 c}}}{\sigma_{e f f}}\left[\frac{1}{s} \int d y_{12} \int d M_{12}^{2} g\left(\overline{x_{1}}, \mu^{2}\right) g\left(\overline{x_{2}}, \mu^{2}\right) \sigma_{g_{1} g_{2} \rightarrow c \bar{c}}\right] \\
& \times\left[\frac{1}{s} \int d y_{34} \int d M_{34}^{2} g\left(\overline{x_{3}}, \mu^{2}\right) g\left(\overline{x_{4}}, \mu^{2}\right) \sigma_{g_{3} g_{4} \rightarrow c \bar{c}}\right] \\
& \times \Theta\left(1-\overline{x_{1}}-\overline{x_{3}}\right) \Theta\left(1-\overline{x_{2}}-\overline{x_{4}}\right) \Theta\left(M_{12}^{2}-4 m_{c}^{2}\right) \Theta\left(M_{34}^{2}-4 m_{c}^{2}\right) \\
& \times \delta\left(y_{34}-y_{12}\right)
\end{aligned}
$$

where

$$
\overline{x_{1}}=\frac{M_{12}}{\sqrt{s}} e^{y_{12}} \quad, \quad \overline{x_{2}}=\frac{M_{12}}{\sqrt{s}} e^{-y_{12}} \quad, \quad \overline{x_{3}}=\frac{M_{34}}{\sqrt{s}} e^{y_{34}} \quad, \quad \overline{x_{4}}=\frac{M_{34}}{\sqrt{s}} e^{-y_{34}}
$$

and consequently

$$
\Theta\left(1-\overline{x_{1}}-\overline{x_{3}}\right)=\Theta\left(1-\frac{M_{12}}{\sqrt{s}} e^{y_{12}}-\frac{M_{34}}{\sqrt{s}} e^{y_{34}}\right),
$$




$$
\Theta\left(1-\overline{x_{2}}-\overline{x_{4}}\right)=\Theta\left(1-\frac{M_{12}}{\sqrt{s}} e^{-y_{12}}-\frac{M_{34}}{\sqrt{s}} e^{-y_{34}}\right)
$$

From the above expressions it is easy to see that when $y_{12}=y_{34}=y$, then (5) holds and the theta functions give lower and upper limits for the integration in $y$ :

$$
-\ln \frac{\sqrt{s}}{M} \leq y \leq \ln \frac{\sqrt{s}}{M}
$$

The upper limit of $M_{12}$ and $M_{34}$ can be fixed imposing constraints on their sum, $M$. In the case of the $X(3872)$ we already know the mass of the state that we want to produce. In principle we could just use (5) with a fixed value of $M$. However, following the spirit of the CEM, we will assume that when the system with mass $M=M_{12}+M_{34}$ goes to the final state with mass $M_{T}$ it can absorb or emit soft gluons to neutralize color. These gluons carry an energy going from almost zero to the QCD scale, given by $\Delta=O\left(\Lambda_{Q C D}\right)$. Then, from (5) and (6) we have:

$$
M^{\text {min }}=M_{12}^{\min }+M_{34}^{\min }=M_{T}-\Delta
$$

and

$$
M^{\max }=M_{12}^{\max }+M_{34}^{\max }=M_{T}+\Delta
$$

From these equations we can see that, knowing the mass of the tetraquark state and fixing the amount of energy which can be exchanged in the formation of the final state, we constrain the limits in the integrations over $M_{12}$ and $M_{34}$. In the symmetric case of $T_{4 c}$ production $M_{12}^{\min }=M_{34}^{\min }, M_{12}^{\max }=M_{34}^{\max }$, (14) and (15) completely fix these limits. In the case of the $X(3872)$, we may have different choices for $M_{12}^{\min }\left(M_{12}^{\max }\right)$ and $M_{34}^{\min }\left(M_{34}^{\max }\right)$ but they will be correlated.

For the partonic cross section we use the standard leading order QCD result [23]:

$$
\sigma_{g g \rightarrow c \bar{c}}=\frac{\pi \alpha_{s}^{2}\left(m^{2}\right)}{3 m^{2}}\left\{\left(1+\frac{4 m_{c}^{2}}{m^{2}}+\frac{m_{c}^{4}}{m^{4}}\right) \ln \left[\frac{1+\beta}{1-\beta}\right]-\frac{1}{4}\left(7+\frac{31 m_{c}^{2}}{m^{2}}\right) \beta\right\}
$$

with

$$
\beta=\left[1-\frac{4 m_{c}^{2}}{m^{2}}\right]^{1 / 2}
$$

where $m^{2}$ is equal to $M_{12}^{2}$ or $M_{34}^{2}$.

Notice that we used the elementary cross section for heavy quark production (16) also in light quark production. Since this expression only holds for heavy enough quarks, its use here is questionable. In spite of this uncertainty, the existing experience in the literature is encouraging. In [24] the authors used (16) to compute the cross section of strange particle production and calculated the asymmetries in the production of $K^{+} / K^{-}, \Lambda / \bar{\Lambda}$, ...etc. They have used the convolution formula of the parton model and have taken the strange quark mass to be $m_{s} \simeq 500 \mathrm{MeV}$. They could reproduce well the existing data on asymmetries. In our case the invariant mass $M_{34}$ defines the perturbative QCD scale and hence we must have $M_{34}>1 \mathrm{GeV}$. This can be achieved with the light quarks having masses close to zero and transverse momenta in the few $\mathrm{GeV}$ region. Since we are using the one-dimensional version of the CEM, instead of transverse momentum we will assign an effective mass to the light quarks, $m_{q}=0.5 \mathrm{GeV}$, which garantees that $M_{34}>2 m_{q}>1 \mathrm{GeV}$. Moreover, choosing $N_{f}=2$ and $\Lambda_{Q C D}=200 \mathrm{MeV}$, we have typically:

$$
\alpha_{s}=\frac{12 \pi}{\left(33-2 N_{f}\right) \ln \left(\frac{\left(2 m_{q}\right)^{2}}{\Lambda_{Q C D}^{2}}\right)} \simeq 0.4
$$


Although we may expect significant corrections, this number is still small enough for perturbation theory to make sense.

In order to o estimate the nuclear effects on the cross section we have evaluated equation (10) replacing $g\left(x, \mu^{2}\right)$ in the proton by the existing nuclear parton distribution functions based on a global fit of the nuclear data using the DGLAP evolution equations, proposed by Eskola et al. [20], by de Florian and Sassot [21], and the recent one proposed by Eskola et al. [22]. In what follows they will be called EKS, DS, and EPS09, respectively. We checked the consistence among them calculating the nuclear modification factor $R_{A}$, given by:

$$
R_{A}=\frac{x g_{A}}{A g_{p}}
$$

\section{Numerical Results and Discussion}

\section{$4.1 X(3872)$}

We show firstly our results for proton proton scattering in Fig. ??, and after that we present the rising of the cross section with the atomic number.

All results were calculated with $m_{c}=1.2 \mathrm{GeV}$ and $m_{c}=1.3 \mathrm{GeV}$. We choosed $\Delta \approx \Lambda_{Q C D} \approx 200$ $\mathrm{MeV}$ and the constant $F_{X}$ was adjusted so that the central value of the cross section at $\sqrt{7}=7 \mathrm{TeV}$ (shown with a star) corresponds to $\sigma_{X}=30 \mathrm{nb}$ [25]. With all the numbers fixed at the lower energy the energy dependence is given by the model.
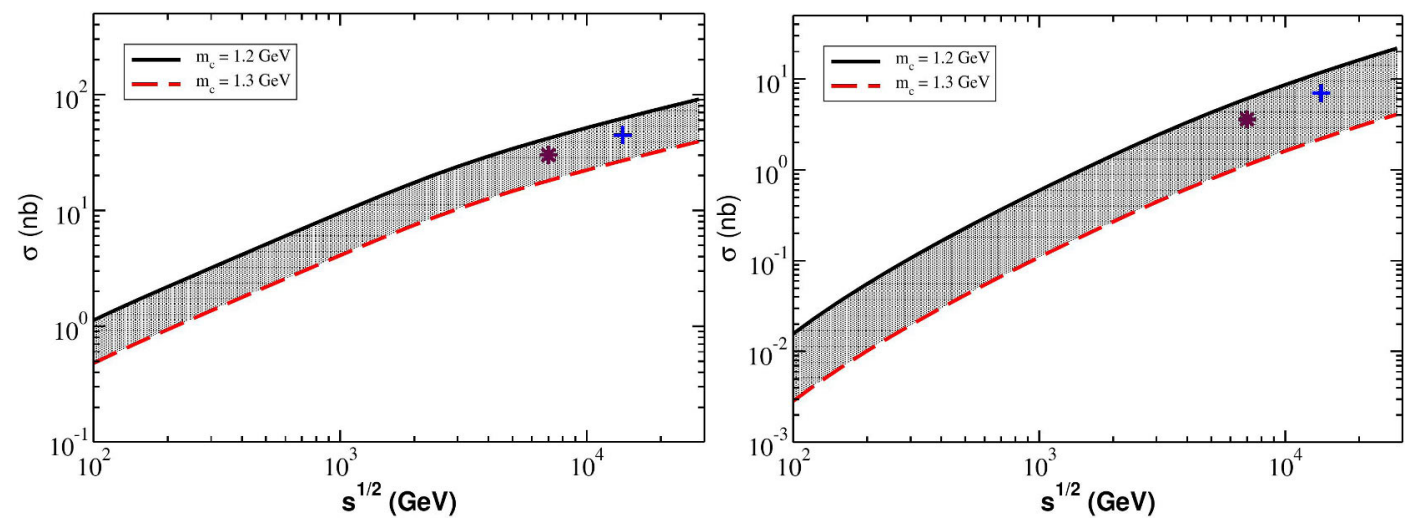

Figure 2. Ratios

We found that the production cross section grows rapidly with the collision energy $\sqrt{s}$ and we are able to make predictions for the forthcoming higher energy data of the LHC.

In Fig. 3 we present our comparison of the shadowing produced by the different nuclear parton distribution functions:

As we can see in Fig. 3, while the DS parametrization predicts a small value of shadowing, the EKS and EPS one predict a similar and large amount of shadowing. The discrepancies among the parton distribution functions imply an extra uncertainty in the predictions.

Then we present our results for X(3872) (Fig. 4-a ) and for T4c (Fig. 4b) production in proton-lead scattering. 


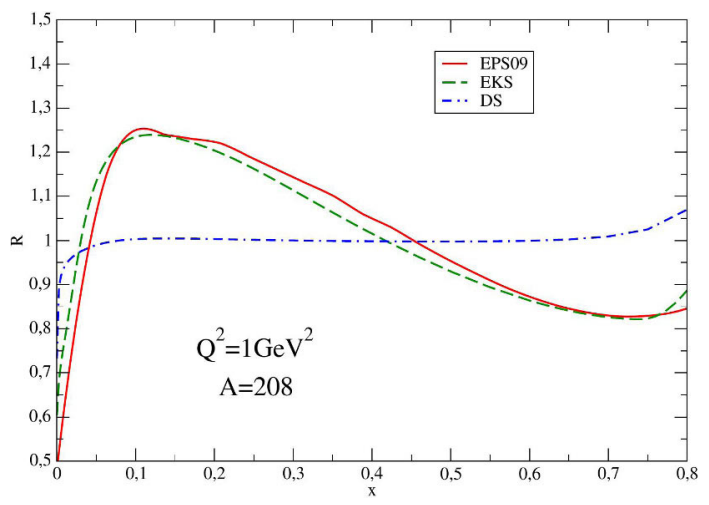

Figure 3. Nuclear modification factor for different parton distribution functions
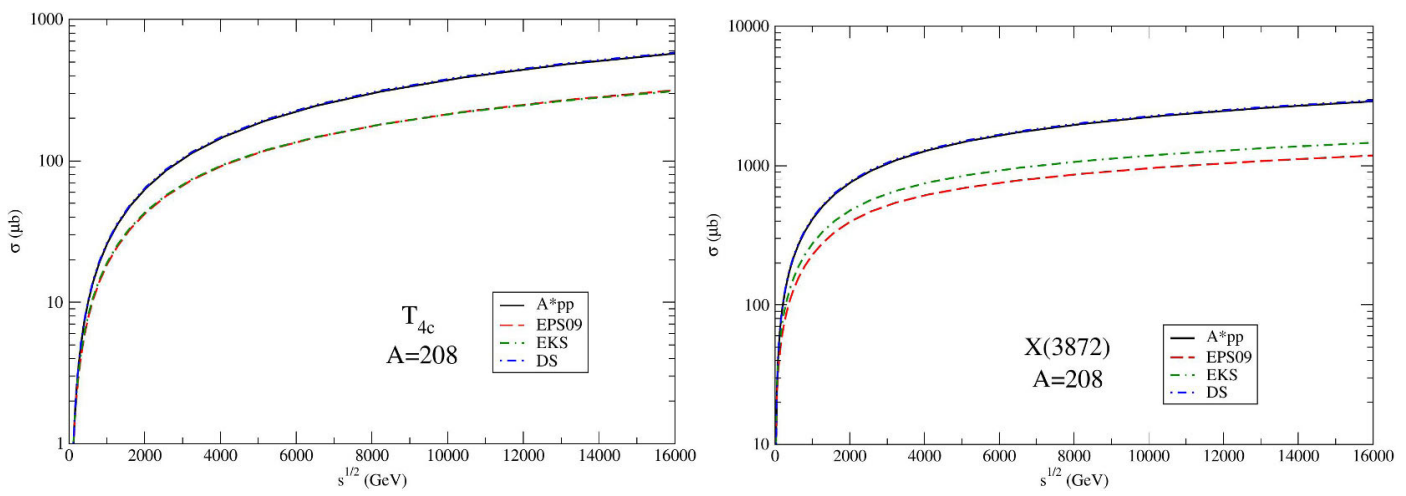

Figure 4. The production cross section for Exotic mesons production in $p A$ collisions.

To summarize we have developed a model for tetraquark production in $\mathrm{pp}$ and $\mathrm{pA}$ collisions which combines double parton scattering and the basic ideas of the color evaporation model. We have made predictions for the $\mathrm{T} 4 \mathrm{c}$ production cross sections and also for $\mathrm{X}(3872)$ at higher energies, which may be confronted with the forthcoming LHC data taken at $\sqrt{7} \mathrm{TeV}$.

We have also applied the model in proton-nucleus collisions and found a rapid growth with the atomic number, despite the different predictions given by the nuclear gluon distribution functions, indicating that tetraquark states can be easily observed in pA collisions.

We are awared that our results contain some uncertainties: (i) They do not include tetraquark production in SPS events; (ii) The binding mechanism is probably too simple and insensitive to the quantum numbers of the involved particles; (iii) In the case of X(3872) production, the use of the elementary cross section for light quark production is questionable; (iv) The differences among the nuclear parton distribution functions;

Despite that, our model is able to predict the behaviour of the cross section with the energy and estimate the size of the nuclear effects on exotic mesons production. 


\section{ACKNOWLEDGMENTS}

This work was supported by São Paulo Research Foundation (FAPESP), grant number 2016/10001-2

\section{References}

[1] Journal Author, Journal Volume, page numbers (year)

[2] Book Author, Book title (Publisher, place, year) page numbers

[3] A. Esposito, A. L. Guerrieri, F. Piccinini, A. Pilloni and A. D. Polosa, Int. J. Mod. Phys. A 30, 1530002 (2014).

[4] Y. Iwasaki, Prog. Theor. Phys. 54, 492 (1975).

[5] J.P. Ader, J.-M. Richard, and P. Taxil, Phys. Rev. D 25, 2370 (1982); J.L. Ballot and J.-M. Richard, Phys. Lett. B 123, 449 (1983); H.J. Lipkin, Phys. Lett. B 172, 242 (1986).

[6] L. Heller and J.A. Tjon, Phys. Rev. D 32, 755 (1985); ibid 35, 969 (1987).

[7] B. Silvestre-Brac, Phys. Rev. D 46, 2179 (1992).

[8] B. Silvestre-Brac and C. Semay, Z. Phys. C 57, 273 (1993); ibid 59, 457 (1993); ibid 61, 271 (1994).

[9] R. J. Lloyd and J. P. Vary, Phys. Rev. D 70, 014009 (2004).

[10] N. Barnea, J. Vijande and A. Valcarce, Phys. Rev. D 73, 054004 (2006).

[11] W. Heupel, G. Eichmann and C. S. Fischer, Phys. Lett. B 718, 545 (2012).

[12] M. Wagner, A. Abdel-Rehim, C. Alexandrou, M. Dalla Brida, M. Gravina, G. Koutsou, L. Scorzato and C. Urbach, J. Phys. Conf. Ser. 503, 012031 (2014).

[13] P. Bicudo, K. Cichy, A. Peters, B. Wagenbach and M. Wagner, Phys. Rev. D 92, 014507 (2015).

[14] A. V. Berezhnoy, A. K. Likhoded, A. V. Luchinsky and A. A. Novoselov, Phys. Rev. D 84, 094023 (2011).

[15] A. V. Berezhnoy, A. V. Luchinsky and A. A. Novoselov, Phys. Rev. D 86, 034004 (2012).

[16] R. Astalos et al., "Proceedings of the Sixth International Workshop on Multiple Partonic Interactions at the Large Hadron Collider," arXiv:1506.05829 [hep-ph]; M. Diehl, PoS DIS 2013, 006 (2013); [arXiv:1306.6059 [hep-ph]].

[17] For recent reviews see S. Bansal et al., arXiv:1410.6664 [hep-ph]; A. Szczurek, Acta Phys. Polon. Supp. 8, 483 (2015); Acta Phys. Polon. B 46, 1415 (2015).

[18] S. Chatrchyan et al. [CMS Collaboration], JHEP 1304, 154 (2013).

[19] F. Carvalho, E.R. Cazaroto , V.P. Goncalves and F.S. Navarra, Phys. Rev. D 93, 034004 (2016).

[20] K. J. Eskola, V. J. Kolhinen, C. A. Salgado, Eur. Jour. Phys. C 9, 61, (1999).

[21] D. de Florian, R. Sassot, Phys. Rev. D 69, 074028 (2004).

[22] K. J. Eskola, H. Paukkunen, C. A. Salgado, J HEP 0807, 102, (2008).

[23] R. Vogt, Ultrarelativistic Heavy Ion Colisions, Elsevier (2007), pg. 396.

[24] T. D. Gutierrez and R. Vogt, Nucl. Phys. A 705, 396 (2002).

[25] S.Chatrchyanetal, [CMS Collaboration], J. HEP 1304,154(2013). 